\title{
Microbiological analysis of contact lens cases and effective health behaviors
}

\author{
Fatemeh Eslami $^{\circledR}$, Hamid Reza Ghasemi Basir $^{2 *}$, Abbas Moradi $^{(}{ }^{\circledR}$, Mina Bayat $^{3}{ }^{\circledR}$ \\ 'Department of Ophthalmology, School of Medicine, Hamadan University of Medical Sciences, Hamadan, Iran \\ ${ }^{2}$ Department of Pathology, School of Medicine, Hamadan University of Medical Sciences, Hamadan, Iran \\ ${ }^{3}$ School of Medicine, Hamadan University of Medical Sciences, Hamadan, Iran
}

*Correspondence to

Hamid Reza Ghasemi Basir, Email: hrgb2004@

yahoo.com, ha.ghasemi@

umsha.ac.ir

Received 11 May 2020 Accepted 10 Aug. 2020 Published online 6 Sep. 2020

Keywords: Contact lens case, Microbiological analysis, Health behaviors

\section{Abstract}

Introduction: Contact lenses are increasingly being used for cosmetic or therapeutic purposes, followed by subsequent contamination and complications such as keratitis. The lens case is one of the most common places to find the cause of contamination.

Objectives: This study was designed to evaluate the health behaviors affecting the lens case contamination and its relationship with the result of lens case culture which can help in prevention of complications.

Patients and Methods: In this cross-sectional study that was performed in northwest of Iran, 150 asymptomatic participants were assessed for health behaviors affecting the lens case contamination and their lens cases were sampled for culture and antibiogram. Data were analyzed with SPSS-16 software.

Results: The frequency of positive microbial culture in medical and cosmetic contact lens cases was $30.7 \%$ and $66.8 \%$, respectively and $32.7 \%$ in general. Among the isolated bacteria observed in positive cultures, Alcaligenes, Enterobacter aerogenes, gram-positive Diphtheroid bacilli, Pseudomonas aeruginosa and Staphylococcus epidermidis were the most common microorganisms, respectively.

Conclusion: A significant proportion of contact lens cases, especially those used for cosmetic purposes had bacterial contamination. Failure to replace the lens case for more than 9 months and the mismatch of the lens solution brand with its storage case will increase bacterial contamination. Washing the lens case with soap and water, and drying it after washing, will reduce bacterial contamination.
Citation: Eslami F, Ghasemi Basir HR, Moradi A, Bayat M. Microbiological analysis of contact lens cases and effective health behaviors. Immunopathol Persa. 2021;7(2):e17. DOI: $10.34172 /$ ipp. 2021.17

\section{Introduction}

Today, contact lenses are increasingly being used for cosmetic or therapeutic purposes. Daily wear of contact lenses requires the use of a contact lens storage case in which to store and disinfect contact lenses overnight. During contamination of contact lenses, the contact lens case becomes a potential source of microorganisms, especially when it is not being properly disinfected (1). Microbial contamination of a contact lens case can compromise contact lens wear and lead to lens-related adverse events. Further, identical strains of bacteria have been isolated from both the corneal ulcer in microbial keratitis and the lens storage case (2). Observation of certain hygiene principles, such as washing the hands before using a lens, drying the lens case, using a lens case disinfectant and more, may reduce lens and lens case contamination (2). Smear, culture and antibiogram are needed to diagnose and treat patients who are involved by complications of lens contamination that among them, the lens case is one of the

\section{Key point}

A significant proportion of contact lens cases had bacterial contamination which is related to health behaviors of users.

most common places to find the cause of contamination. Investigating the effect of hygienic principles and factors affecting the lens case contamination and its relationship with the result of lens case culture can help in prevention of complications.

Contact lenses are used for both therapeutic and cosmetic purposes. Contact lenses are considered medical devices and lens quality assessment is performed by the Food and Drug Administration (FDA) (3). These lenses should be rinsed and soaked in a special lens case with disinfectant solutions, according to a daily schedule (4). It must be noted that lens contamination occurs in more than $85 \%$ of cases, which is hidden from users and does not lead to signs and symptoms $(5,6)$.

\footnotetext{
Copyright $(\subset 2021$ The Author(s); Published by Nickan Research Institute. This is an open-access article distributed under the terms of the Creative Commons Attribution License (http://creativecommons.org/licenses/by/4.0), which permits unrestricted use, distribution, and reproduction in any medium, provided the original work is properly cited.
} 
Previous studies have shown that lens case contamination is common and ranges from 30 to $80 \%(5-8)$.

The use of contact lenses may be associated with itchy, foreign body sensation in the eyes, and redness, which approximately one-third of contact lens users have experienced at least once (9). More than $99 \%$ of contact lens users are at least, once at risk for eye infection (10). Poor hygiene of contact lenses causes microbial keratitis (11) and corneal damage, depends more on the frequency of keratitis than the severity of the infection (12). Corneal ulcer and visual loss due to the use of contact lenses may require a corneal transplantation. In developed countries, more than $30 \%$ of keratitis cases are caused by contact lenses (13).

Contact lens contamination may occur when a person uses the lens, or the contamination may be due to the lens case as a reservoir for microorganisms (2). The risk of infection will be decreased by drying the environment in which we put the lenses. The position of the lens case during drying is effective in contamination; therefore, the face down condition results in reduction in microbial contamination (14). Risk factors of eye infections due to using of contact lenses include; sleeping with contact lenses, swimming and showering with contact lenses, cleaning and keeping lenses in tap water, no using of the lens case, late lens case replacement, long-term use of the lens, and lack of attention to the lens expiration date (10), no washing hands with soap before putting on and taking off the lenses, and mismatch within the lens case and lens solution brands (2). The main function of lens case solution is to clean and disinfect. The "cleaning" is to remove protein and lipid and the "disinfection" is to substantially decrease microbial load (15). In addition, multipurpose disinfectants such as $\mathrm{H}_{2} \mathrm{O}_{2} 3 \%$ can also eliminate cyst and trophozoites (7). It is best to clean the lens case daily with a disinfectant liquid and replace it every three months (10). Cleaning the lens case with tap water will increase contamination of the lenses with gramnegative bacteria. In addition, if the case is changed every two weeks, we will have the least amount of contamination (2). Bacterial agents, especially Staphylococcus epidermis and Pseudomonas aeruginosa, are the most common contact lens contaminants. Fungal agents such as Candida and Cladosporium, and finally protozoa such as Acanthamoeba are in the next category $(7,13,16)$. Bacillus, Propionibacterium and Corynebacterium species have also been reported from the lens case cultures (2).

\section{Objectives}

This study was designed to determine the common regional microorganisms contaminating the lens case, and their antibiotic susceptibility results, as well as its relationship to the health behaviors of asymptomatic contact lens wearers. Findings of this study can be helpful in determination of the probable pathogens and the effective antibiotics which can be used in empirical therapy of symptomatic patients involved by lens-related adverse events, before preparing the result of corneal ulcer culture and antibiogram.

\section{Patients and Methods \\ Study design}

This cross-sectional study was performed at the eye clinic of Farshchian hospital in Hamadan, located in northwest of Iran, in 2017, using the convenience sampling method. The sample size of 150 was calculated using Rahim et al. study (17) based on the formula used in descriptive qualitative studies, according to the prevalence of positive culture result of lens case $(\mathrm{P}=89 \%)$ with $5 \%$ alpha and $5 \%$ sampling error.

In this study, asymptomatic contact lens wearers who had at least three months history of lens use experience were called, and after explaining the study conditions and obtaining written consent, were referred to the laboratory for lens case culture, until completing sample size. Age over 18 years and absence of apparent ocular pathology in slit lamp examination were regarded as inclusion criteria. The presence of clinical signs or symptoms and reluctance to performing lens case culture were considered as exclusion criteria. Contact lens hygiene behavior questionnaires were delivered after recruiting and composed of hand washing, method of hand washing, washing the lens case, type of liquid used to wash the case and method of drying the case.

The lens case was sampled for culture using sterile swab and normal saline, in four culture medias (thioglycollate broth, blood agar, chocolate agar and eosin methylene blue), under aseptic conditions. Culture media were assessed after 48 hours incubation at $37^{\circ} \mathrm{C}$. In addition, differential culture media were used to accurately diagnose the type of bacteria, as well as antibiogram discs (Mast, UK) appropriate to the cultured microorganism. The results of the antibiogram were interpreted based on the Clinical and Laboratory Standards Institute (CLSI) 2017. Results of culture and antibiogram and other data including age, gender, education (high school and diploma, undergraduate and postgraduate), contact lens type (therapeutic lens for correction of refractive errors or cosmetic lens which has no lens power and use purely for cosmetic purposes to change eye color), lens case brand, lens solution brand (commercial mark available in market which is sold with the case), duration of contact lens experience according to medical records, lens replacement time interval, how to wash hands when placing the lens, method of washing the lens case and method of drying the lens case were asked and recorded in the checklist.

\section{Ethical issues}

The research followed the tenets of the Declaration of Helsinki. The Ethics Committee of Hamadan University of Medical Sciences approved this study. The institutional ethical committee at Hamadan University of Medical Sciences approved all study protocols (IR.UMSHA. 
REC.1396.793). Accordingly, written informed consent was taken from all participants before any intervention. This study was extracted from the MD thesis of Mina Bayat at this university (Thesis\#9611247620).

\section{Data analysis}

Data were analyzed after completion with SPSS-16 software. Descriptive and qualitative data were expressed as tables, graphs, ratios and percentages. Central and distribution indices were used to summarize quantitative variables. To compare the results of lens cases culturing in terms of qualitative, nominal and ranking variables, chi-square test (or Fisher's exact test), and in terms of quantitative variables, student t-test (or nonparametric Mann-Whitney-U test) was used. The significance level was considered $<0.05$.

\section{Results}

In this study which was conducted to determine the degree of contamination of contact lens cases and the factors affecting it, 150 people using contact lenses along with 150 relevant lens cases were assessed. The mean age and standard deviation of peoples were $28.43 \pm 6.68$ years, and in terms of gender, 30 (20\%) were male and 120 (80\%) were female. The prevalence of positive bacterial culture in the examined lens cases was 49 cases (32.67\%).

The mean age and standard deviation of people with lens case contamination (positive bacterial culture with any colony count) was $28.44 \pm 6.01$ years and those without contamination (negative bacterial culture) were $28.40 \pm 6.56$ years. Based on the results of the $t$ test, no statistically significant difference was observed between the two groups in terms of age $(P=0.970)$.

In 42 subjects (35\%) of the 120 women and in 7 subjects (23.3\%) of the 30 men, the results of lens case culture were positive. Based on the results of chi-square test, no statistically significant difference was observed between males and females in terms of culture results $(P=0.194)$.

Despite the high percentage of positive culture in people with high school and diploma (39.1\%), and the low percentage in people with undergraduate $(32.1 \%)$ and postgraduate $(26.9 \%)$ educations, due to the high level of education in most participants and based on Fisher's exact test, there was no statistically significant difference between the level of education and the result of lens case culture $(P=0.615)$.
The mean and standard deviation of the duration of contact lens experience in people with and without positive lens case culture were $46.52 \pm 40.05$ months and $49.94 \pm 36.44$ months, respectively. Based on the results of Mann-Whitney U test, no statistically significant difference was observed between the duration of experience and the result of the lens case culture $(P=0.452)$.

The mean and standard deviation of lens case replacement time in people with positive and negative culture results were $10.10 \pm 4.77$ months and $6.95 \pm 4.92$ months, respectively. According to the findings in Table 1 , the lowest contamination rate of the lens case was in people who changed the lens case in less than three months apart (2.6\%). Most of contamination rates were in lens case replacements between 9 to 12 months (48.2\%) and more than 12 months (50\%). Based on the results of Mann-Whitney U test, a significant statistical correlation was observed between the time interval of lens case replacement and the result of lens case culture $(P<0.001)$, i.e. the rate of contamination increases with increasing time interval.

The percentage of positive culture results, in cases of matching and mismatching of the lens case and lens solution brands, was $18.37 \%$ and $60.8 \%$, respectively. Based on the results of chi-square test, the percentage of positive culture results was significantly higher in lenscase/lens-solution mismatch cases $(P<0.001)$.

The percentage of positive culture results in therapeutic and cosmetic contact lenses were $30.7 \%$ and $66.7 \%$, respectively. Based on Fisher's exact test, the percentage of positive results of culture in cosmetic contact lens users was significantly higher than therapeutic type $(P=0.038)$.

According to the findings of Table 2, no significant statistical relationship was observed between the positive result rate of lens-case culture and hand washing, method of hand washing and washing or non-washing of the lens case. However, the type of liquid used to wash the case and also drying the case were significantly associated with reducing the bacterial contamination of the lens case (Figure 1).

Among the bacteria observed in lens case cultures, Alcaligenes and Enterobacter aerogenes with 20.41\% and gram-positive Diphtheroid bacilli and Pseudomonas aeruginosa with $18.37 \%$ and finally Staphylococcus epidermidis with $14.28 \%$ frequency, were the most common isolated microorganisms respectively (Figure 2).

Table 1. Frequency of lens case contamination according to the time interval between lens case replacement

\begin{tabular}{|c|c|c|c|c|}
\hline \multirow{3}{*}{$\begin{array}{l}\text { Time interval between lens case } \\
\text { replacement }\end{array}$} & \multicolumn{2}{|c|}{ Microbial Culture Result } & \multirow{3}{*}{ Total } & \multirow{3}{*}{$P$ value } \\
\hline & Positive & Negative & & \\
\hline & No. $(\%)$ & No. $(\%)$ & & \\
\hline Less than 3 months & $1(2.6 \%)$ & $37(97.4 \%)$ & 38 & \multirow{4}{*}{$<0.001$} \\
\hline 3-9 months & $18(36 \%)$ & $32(64 \%)$ & 50 & \\
\hline More than 9 months & $30(48.4 \%)$ & $32(51.6)$ & 62 & \\
\hline Total & $49(32.66 \%)$ & $101(67.33 \%)$ & 150 & \\
\hline
\end{tabular}


Table 2. The frequency of lens case contamination in contact lens users depending on how to wash the hand and case, the type of detergent and the method of drying the case

\begin{tabular}{|c|c|c|c|}
\hline \multirow[b]{2}{*}{ Individual health behavior } & \multicolumn{2}{|c|}{ Microbial Culture Result } & \multirow[b]{2}{*}{$P$ value } \\
\hline & $\begin{array}{c}\text { Positive } \\
(n=49)\end{array}$ & $\begin{array}{c}\text { Negative } \\
(n=101)\end{array}$ & \\
\hline \multicolumn{4}{|l|}{ Washing the hands } \\
\hline Yes & $47(95.9 \%)$ & $101(100 \%)$ & \multirow{2}{*}{$0.105^{*}$} \\
\hline No & $2(4.1 \%)$ & $0(0 \%)$ & \\
\hline \multicolumn{4}{|l|}{ Washing the hands with } \\
\hline Water alone & $1(2.1 \%)$ & $1(1 \%)$ & \multirow{3}{*}{$0.389^{*}$} \\
\hline Soap and water & $46(95.8 \%)$ & $98(98 \%)$ & \\
\hline Disinfectant solution and water & $1(2.1 \%)$ & $1(1 \%)$ & \\
\hline \multicolumn{4}{|l|}{ Washing the lens case } \\
\hline Yes & $43(87.8 \%)$ & $94(93.1 \%)$ & \multirow{2}{*}{$0.354^{* *}$} \\
\hline No & $6(12.2 \%)$ & $7(6.9 \%)$ & \\
\hline \multicolumn{4}{|l|}{ Washing the lens case with } \\
\hline Water alone & $17(37.8 \%)$ & $14(15.2 \%)$ & \multirow{4}{*}{$0.027^{*}$} \\
\hline Soap and water & $18(40 \%)$ & $56(60.9 \%)$ & \\
\hline Disinfectant solution and water & $8(17.8 \%)$ & $19(20.7 \%)$ & \\
\hline Lens case solution & $2(4.4 \%)$ & $3(3.2 \%)$ & \\
\hline \multicolumn{4}{|l|}{ Drying the lens case } \\
\hline Yes & $12(24.5 \%)$ & $48(47.5 \%)$ & \multirow{2}{*}{$0.008^{* *}$} \\
\hline No & $37(75.5 \%)$ & $53(52.5 \%)$ & \\
\hline
\end{tabular}

*Fisher's exact test, ** Chi-square test.

According to Table 3, Alcaligenes microorganism had the highest susceptibility to ceftazidime (80\%), clindamycin (70\%), and ciprofloxacin (60\%). Enterobacter aerogenes showed the highest susceptibility to ciprofloxacin (100\%), gentamicin (90\%), and ceftazidime (80\%). Additionally, Pseudomonas aeruginosa had the highest susceptibility to ceftazidime (77.78\%) and ciprofloxacin (77.78\%). Staphylococcus epidermidis also showed the highest susceptibility to co-trimoxazole (100\%), clindamycin
(85.71\%), and ciprofloxacin (71.43\%), and its highest antibiotic resistance was seen against vancomycin (85.71\%) and ceftazidime (57.14\%).

In addition, Stenotrophomonas Maltophilia isolated from the contact lens case had the highest susceptibility to gentamicin (50\%) and ciprofloxacin (50\%) and was $100 \%$ resistant to ampicillin, clindamycin and cephalothin.

One case of Escherichia coli was observed among positive cultures, which was susceptible to ampicillin, ceftazidime, clindamycin, ciprofloxacin, and gentamicin. One case of isolated Listeria was also susceptible to vancomycin, clindamycin, ciprofloxacin, and gentamicin, and resistant to ampicillin and cefazolin. In addition, all gram-positive Diphtheroid bacilli isolated from the lens case were fully susceptible to all antibiotics including co-trimoxazole, ceftazidime, clindamycin, ciprofloxacin, gentamicin, and vancomycin.

\section{Discussion}

In the current study, the frequency of positive microbial culture in therapeutic and cosmetic contact lens cases was $30.7 \%$ and $66.8 \%$, respectively and $32.7 \%$ in general. Among the isolated bacteria observed in positive cultures, Alcaligenes, Enterobacter aerogenes, grampositive Diphtheroid bacilli, Pseudomonas aeruginosa and Staphylococcus epidermidis were the most common microorganisms, respectively.

The study of Anitha et al, to evaluate the rate of bacterial contamination of contact lenses among medical students in India, $88 \%$ of samples showed positive microbial culture and the most common contaminants were Pseudomonas aeruginosa (23\%), Staphylococcus aureus (18\%), E. coli (16\%), Citrobacter koseri (11\%), Acinetobacter baumannii

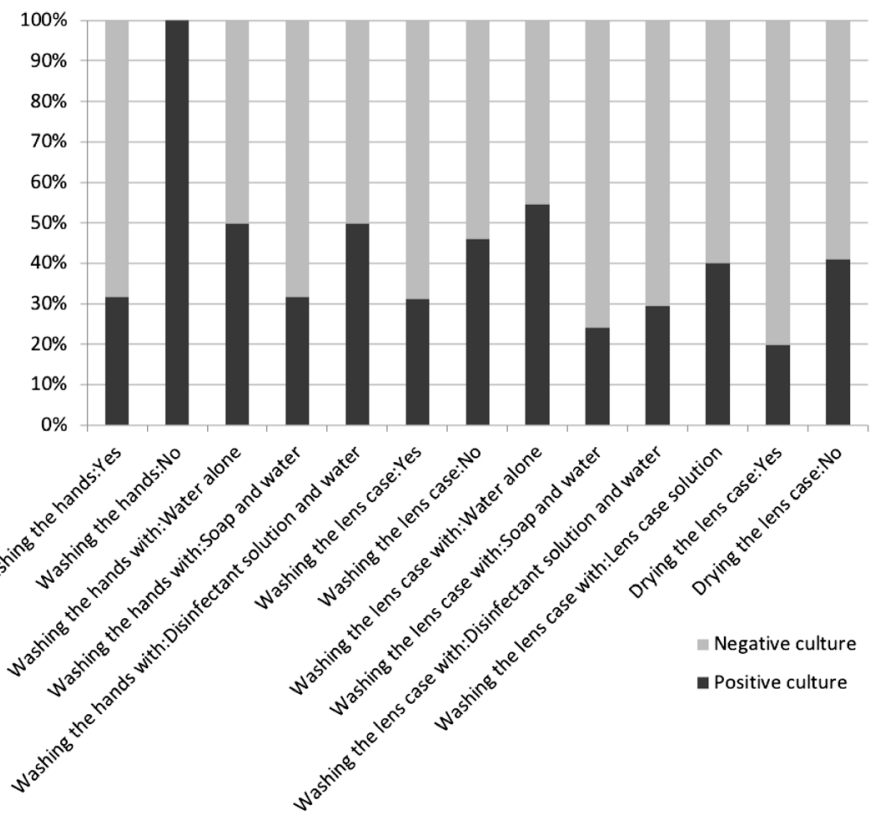

Figure 1. The frequency of lens case contamination in contact lens users depending on how to wash the hand and case, the type of detergent and the method of drying the case. 


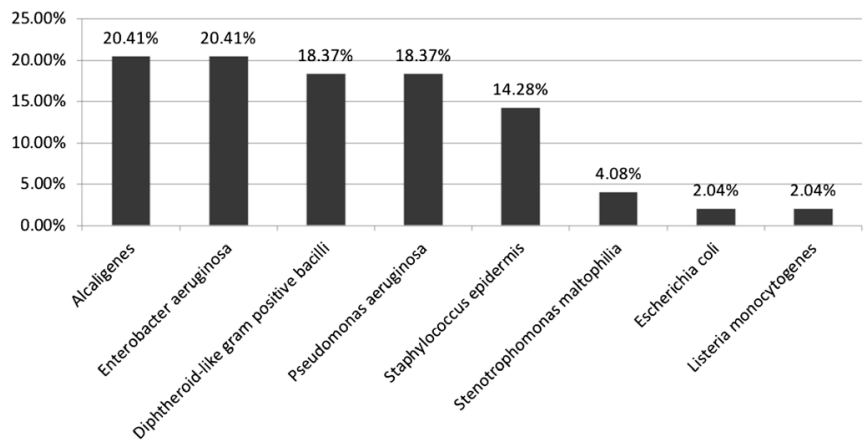

Figure 2. The frequency of bacterial microorganisms in the results of positive culture of lens cases.

(11\%), and Klebsiella pneumoniae (9\%), Micrococcus (7\%) and Proteus (5\%), respectively (18). In a study conducted by Cope et al in the United States on 1075 cases, the highest prevalence of contamination was reported in daily soft contact lenses $(57.2 \%)$, contact lenses for long hours consumption (35.5\%) and rigid gas permeable lenses (4\%), respectively (10).

In the study conducted by Wu et al in Australia, lens

Table 3. Frequency of antibiotic susceptibility and resistance of microorganisms isolated from lens cases

\begin{tabular}{|c|c|c|c|c|}
\hline \multirow{2}{*}{ Antibiotic/Microorganism } & \multicolumn{3}{|c|}{ Antibiogram Result, No. (\%) } & \multirow{2}{*}{ Total } \\
\hline & Susceptible & Intermediate & Resistance & \\
\hline \multicolumn{5}{|l|}{ Ampicillin } \\
\hline Alcaligenes & $0(0)$ & $2(20)$ & $8(80)$ & 10 \\
\hline Enterobacter aeruginosa & $0(0)$ & $0(0)$ & $10(100)$ & 10 \\
\hline Pseudomonas aeruginosa & $0(0)$ & $0(0)$ & $9(100)$ & 9 \\
\hline \multicolumn{5}{|l|}{ Ceftazidime } \\
\hline Alcaligenes & $8(80)$ & $1(10)$ & $1(10)$ & 10 \\
\hline Enterobacter aeruginosa & $8(80)$ & $1(10)$ & $1(10)$ & 10 \\
\hline Pseudomonas aeruginosa & $7(77.78)$ & $0(0)$ & $2(22.22)$ & 9 \\
\hline Staphylococcus epidermis & $1(14.29)$ & $2(28.57)$ & $4(57.14)$ & 7 \\
\hline \multicolumn{5}{|l|}{ Ciprofloxacin } \\
\hline Alcaligenes & $6(60)$ & $4(40)$ & $0(0)$ & 10 \\
\hline Enterobacter aeruginosa & $10(100)$ & $0(0)$ & $0(0)$ & 10 \\
\hline Pseudomonas aeruginosa & $7(77.78)$ & $1(11.11)$ & $1(11.11)$ & 9 \\
\hline Staphylococcus epidermis & $5(71.43)$ & $0(0)$ & $2(28.57)$ & 7 \\
\hline \multicolumn{5}{|l|}{ Gentamicin } \\
\hline Alcaligenes & $0(0)$ & $0(0)$ & $10(100)$ & 10 \\
\hline Enterobacter aeruginosa & $9(90)$ & $0(0)$ & $1(10)$ & 10 \\
\hline Pseudomonas aeruginosa & $6(66.67)$ & $0(0)$ & $3(33.33)$ & 9 \\
\hline \multicolumn{5}{|l|}{ Clindamycin } \\
\hline Alcaligenes & $7(70)$ & $1(10)$ & $2(20)$ & 10 \\
\hline Enterobacter aeruginosa & $6(60)$ & $0(0)$ & $4(40)$ & 10 \\
\hline Pseudomonas aeruginosa & $3(33.33)$ & $3(33.33)$ & $3(33.33)$ & 9 \\
\hline Staphylococcus epidermis & $6(85.71)$ & $0(0)$ & $1(14.29)$ & 7 \\
\hline \multicolumn{5}{|l|}{ Cefalotin } \\
\hline Alcaligenes & $0(0)$ & $1(10)$ & $9(90)$ & 10 \\
\hline Enterobacter aeruginosa & $0(0)$ & $0(0)$ & $10(100)$ & 10 \\
\hline Pseudomonas aeruginosa & $1(11.11)$ & $0(0)$ & $8(88.89)$ & 9 \\
\hline \multicolumn{5}{|l|}{ Vancomycin } \\
\hline Staphylococcus epidermis & $1(14.29)$ & $0(0)$ & $6(85.71)$ & 7 \\
\hline \multicolumn{5}{|l|}{ Cotrimoxazole } \\
\hline Pseudomonas aeruginosa & $6(66.67)$ & $0(0)$ & $3(33.33)$ & 9 \\
\hline Staphylococcus epidermis & $7(100)$ & $0(0)$ & $0(0)$ & 7 \\
\hline
\end{tabular}

cases contaminations were reported in $66 \%$ of cases and coagulase-negative Staphylococcus was the most common isolated microorganism (2).

In a study by Thakur et al in India on the rate of microbial contamination in 50 asymptomatic lens users, $74 \%$ of the participants had at least one positive culture sample that among which the sample of lens cases had the highest level of contamination (62\%). While contact lenses, lens solution bottles and lens solutions were contaminated in $56 \%, 48 \%$, and $42 \%$ of cases, respectively and the most common reported microorganisms were Staphylococcus aureus (21\%) and Pseudomonas (19.5\%) (13). In a study by Rahim et al in Pakistan with assessment of 100 pharmacy students, contact lens, lens case and conjunctival contaminations were observed in $65 \%, 89 \%$ and $32 \%$ of the samples, respectively and the most common contaminants were Staphylococcus epidermidis (39.8\%) and Pseudomonas aeruginosa (34.9\%) (17). Additionally, $85 \%$ of samples were taken from solution in contact lens storage cases and $65 \%$ of contact lenses were contaminated in the study of Mohamed et al that was conducted on 30 contact lens wearers in Egypt, and the most common isolated microorganisms were Staphylococcus epidermidis (36.3\%), Pseudomonas aeruginosa (34.7\%), and Staphylococcus aureus (15.3\%), respectively (19).

As can be seen, the range of contamination rates reported in different studies is between $35 \%$ and $89 \%$, and in our study, the lowest level of contamination (32.7\%) was reported. The reason for the inconsistency of results in different studies can be due to differences in sample size (from 30 to 1075 people), differences in sampling location (lens, lens case, lens case solution, conjunctiva), differences in the study population (asymptomatic people, patients with ocular symptoms, students, general population), differences in the type of lens (soft lens, hard lens, therapeutic or cosmetic lens, daily wear, monthly, quarterly or yearly) and the type of health behaviors of people in different communities. These differences are also evident in the type of isolated microorganisms. In general, a significant proportion of contact lenses, lens cases, and preservatives in various studies have microbial contamination. In addition, Staphylococcus epidermidis and Pseudomonas, which were common strains in most 
studies, are also common isolated bacteria in our study.

Due to the differences in the type of isolated microorganisms, and the type of antibiotics that used, the pattern of antibiotic susceptibility and resistance varies in the different studies. However, in our study, ciprofloxacin was one of the most effective antibiotics against most strains isolated from lens cases.

In the study by $\mathrm{Wu}$ et al, streptococcal species, staphylococcal species and Pseudomonas were the most common classes of infective agents in microbial keratitis. Fluoroquinolones have also been used as monotherapy and were highly effective against gram-positive and gram-negative bacteria. In addition, it has been shown that microorganisms isolated from contact lenses that are associated with infectious eye diseases are the same as those isolated from the corresponding contact lens cases (20).

Another notable point is that the range of bacteria contributed to contamination of contact lens cases, are not usually associated with eye diseases correlated to contact lens use, which is suggesting that factors other than lens and lens case contamination are related to infectious keratitis (1).

The results of the present study were consistent with the findings of the study by Thakur et al (13) in terms of higher contamination of cosmetic lens cases than therapeutic types, that may be related to the type of health behaviors and the likelihood of infection in cosmetic users.

In the current study, most users of contact lenses were young and women in terms of age and gender, and had high education; since, there was no statistically significant relationship between contamination rate of the lens case with age, gender and education. The reason may be that the groups are relatively homogeneous in terms of the mentioned variables.

In the present study, no significant statistical relationship was observed between the duration of the experience of using the lens and the positive result of lens case culturing. However, the mean duration of lens case replacement in positive culture group was significantly higher than in negative culture group. The lowest level of contamination was in the replacement of the lens case under three months (2.6\%), and the highest level of contamination was in the replacement more than nine months (48.4\%). In a study by Wu et al (19), in line with the results of the current study, lens cases that were used for less than 9 months had the lowest levels of contamination. Moreover, in a study by Cope et al (10) in the United States, it was recommended to replace the lens case every three months. Increasing the duration of the lens case using and accumulation of unhealthy behaviors during this period can be led to bacterial colonization which intensified in delayed lens case replacement.

In the present study, the percentage of positive culture results, in cases with mismatching of the lens case while, lens solution brands, was significantly higher than matching cases. Consistent with the results of our study, in the study of $\mathrm{Wu}$ et al (2), in $25 \%$ of cases, the lens case and the lens case solution were not from the same brand, in which case the multiplicity and intensity of case contamination was higher. This may be due to the fact that the preservative in the lens solution is more suitable for the lens case of the same brand. This matching may also be seen more in people who follow more hygienic principles.

In a study conducted by Willcox et al, as evaluation of lens case contamination in users of daily silicone contact lenses in Australia on 232 participants, $76 \%$ to $92 \%$ of lens cases were contaminated, depending on the brand. In this study, the rate of contamination with fungi and grampositive bacteria was not significantly different in various brands, but the rate of contamination with gram-negative bacteria in users of lens solution containing primary disinfectant was lower than others (5).

In the present study, there was no statistically significant relationship between hand washing, method of hand washing and washing or non-washing of the lens case with the positive result of lens case culture, which can be justified by high levels of other healthy behaviors in these conditions. However, the type of liquid used to wash the case and drying the case, significantly reduced the bacterial contamination of the lens case, so that, washing the lens case with water alone had the most (54.8\%) and water and soap had the least $(24.3 \%)$ rate of contamination. It was also notable that the contamination rates of the lens case in washing with water and disinfectant solution (29.6\%) and also with the lens solution (40\%) were more than contamination of washing with water and soap. The high level of contamination in users of disinfectant solution was unexpected, which could be due to the low-number of these participants or misuse of disinfectant due to the trust in the name of them. Furthermore, drying the lens case makes the environment unsuitable for bacterial growth and can explain the reason for the significant reduction in lens case contamination following the drying in this study.

In a study by Wu et al (2), as the effect of observing hygienic principles on lens case contamination in contact lens users, twelve different brands of lens case solution were used, and it was finally determined that the contamination rate was not related to the brand of lens case solution. Around $61 \%$ of people used soap and water to wash their hands before using lenses, 35\% used water alone and 5\% used lenses without washing their hands that the level of contamination was significantly lower in the first group. The reason for the inconsistency of the results of the present study with the findings of the study of Wu et al (2), in terms of not observing a significant relationship between hand washing method and microbial contamination, is that in the present study, more than $97 \%$ of people used soap and water to wash their hands, and the number of participants in the other two groups was very small for comparison. 


\section{Conclusion}

In eye clinics, a significant proportion of contact lens cases, especially those used for cosmetic purposes had bacterial contamination. Failure to replace the lens case for more than 9 months and the mismatch of the lens solution brand with its storage case will increase bacterial contamination. Washing the lens case with soap and water, and drying it after washing, will reduce bacterial contamination. The most common isolated microorganisms in positive lens case cultures were Alcaligenes, Enterobacter aerogenes, gram-positive Diphtheroid bacilli, Pseudomonas aeruginosa and Staphylococcus epidermidis. In addition, among common microorganisms isolated from the lens case, the most common antibiotic susceptibility was related to ciprofloxacin in antibiograms. Given that one of the most common causes of corneal ulcers is the use of contact lens, recognizing common microorganisms isolated from the lens storage case in each area and their pattern of antibiotic susceptibility can help ophthalmologists in this regard.

\section{Limitations of the study}

The major limitation of our study is that asymptomatic contact lens wearers over 18 years old were enrolled. We suggest further studies will be investigated in the symptomatic patients in all age groups in the future.

\section{Authors' contribution}

HRGB, FE and MB were the principal investigators of the study. $H R G B, F E$ and $M B$ were included in preparing the concept and design. FE and AM revisited the manuscript and critically evaluated the intellectual contents. All authors participated in preparing the final draft of the manuscript, revised the manuscript and critically evaluated the intellectual contents. All authors have read and approved the content of the manuscript and confirmed the accuracy or integrity of any part of the work.

\section{Conflicts of interest}

The authors declare that they have no competing interests.

\section{Ethical considerations}

Ethical issues (including plagiarism, data fabrication, double publication) have been completely observed by the authors.

\section{Funding/Support}

No internal or external source of funding was available.

\section{References}

1. Bôas VTV, Almeida Júnior GC, Almeida MTG, Gonçalves MS, Coelho LF. Microbiological analysis of contact lens cases: impact of the hospital environment. Arq Bras Oftalmol. 2018;81:371-5. doi:10.5935/0004-2749.20180074.

2. Wu YT, Willcox MD, Stapleton F. The effect of contact lens hygiene behavior on lens case contamination. Optom Vis Sci. 2015;92:167-74. doi: 10.1097/OPX.0000000000000634

3. Zaki M, Pardo J, Carracedo G. A review of international medical device regulations: Contact lenses and lens care solutions. Cont Lens Anterior Eye. 2019;42:136-46. doi: 10.1016/j.clae.2018.11.001
4. Carnt NA, Evans VE, Naduvilath TJ, Willcox MD, Papas $\mathrm{EB}$, Frick KD, et al. Contact lens-related adverse events and the silicone hydrogel lenses and daily wear care system used. Arch Ophthalmol. 2009;127:1616-23. doi: 10.1001/ archophthalmol.2009.313.

5. Willcox MD, Carnt N, Diec J, Naduvilath T, Evans V, Stapleton $\mathrm{F}$, et al. Contact lens case contamination during daily wear of silicone hydrogels. Optom Vis Sci. 2010;87:456-64. doi: 10.1097/OPX.0b013e3181e19eda.

6. Yung MS, Boost M, Cho P, Yap M. Microbial contamination of contact lenses and lens care accessories of soft contact lens wearers (university students) in Hong Kong. Ophthalmic Physiol Opt. 2007;27:11-21. doi: 10.1111/j.14751313.2006.00427.x.

7. Anger C, Lally JM. Acanthamoeba: a review of its potential to cause keratitis, current lens care solution disinfection standards and methodologies, and strategies to reduce patient risk. Eye Contact Lens. 2008;34:247-53. doi: 10.1097/ ICL.0b013e31817e7d83.

8. Tilia D, Lazon de la Jara $\mathrm{P}, \mathrm{Zhu} H$, Naduvilath TJ, Holden BA. The effect of compliance on contact lens case contamination. Optom Vis Sci. 2014;91:262-71. doi: 10.1097/ OPX.0000000000000163.

9. Suchecki JK, Donshik P, Ehlers WH. Contact lens complications. Ophthalmol Clin North Am. 2003;16:471-84. doi: 10.1016/ s0896-1549(03)00056-7.

10. Cope JR, Collier SA, Nethercut H, Jones JM, Yates K, Yoder JS. Risk Behaviors for Contact Lens-Related Eye Infections Among Adults and Adolescents - United States, 2016. Morb Mortal Wkly Rep. 2017;66:841-5. doi: 10.15585/mmwr.mm6632a2.

11. Stapleton F, Naduvilath T, Keay L, Radford C, Dart J, Edwards $\mathrm{K}$, et al. Risk factors and causative organisms in microbial keratitis in daily disposable contact lens wear. PLoS One. 2017;12:e0181343. doi: 10.1371/journal.pone.0181343

12. Szczotka-Flynn L, Lass JH, Sethi A, Debanne S, Benetz BA, Albright $M$, et al. Risk factors for corneal infiltrative events during continuous wear of silicone hydrogel contact lenses. Invest Ophthalmol Vis Sci. 2010;51:5421-30. doi: 10.1167/ iovs.10-5456.

13. Thakur DV, Gaikwad UN. Microbial contamination of soft contact lenses \& accessories in asymptomatic contact lens users. Indian J Med Res. 2014;140:307-9.

14. Wu YT, Zhu H, Willcox M, Stapleton F. Impact of air-drying lens cases in various locations and positions. Optom Vis Sci. 2010;87:465-8. doi: 10.1097/OPX.0b013e3181e172a1.

15. Bhandari M, Hung PR. Habits of contact lens wearers toward lens care in Malaysia. Med J Malaysia. 2012;67:274-7. PMID: 23082416

16. Zahrani S. Bacteria isolated from contact and non contact lens and antibiotic susceptibility patterns of isolated Pseudomonas aeruginosa. Afr J Microbiol Res. 2012;6:7350-6. doi: 10.5897/ AJMR12.1134

17. Rahim N, Bano H, Naqvi S. Bacterial Contamination Among Soft Contact Lens Wearer. Pak J Ophthalmol. 2008;24:93-6.

18. Anitha M, Mathivathani P, Ramya K, Vijay M. Assessment of Bacterial Contamination on Contact Lenses among Medicos. Int J Pharma. 2016;4:1160-5. doi: 10.21276/ijprhs.2016.03.03

19. Mohamed JA, Abdallah SM, Alatrouny AM, Newishy HM. Bacterial interaction among soft contact lens users and lens care solutions with antibiotic suseptibility pattern. Egypt J Hosp Med. 2017;68: 982-990. doi: 10.12816/0038200.

20. Wu YTY, Willcox M, Zhu H, Stapleton F. Contact lens hygiene compliance and lens case contamination: a review. Cont Lens Anterior Eye. 2015;38:307-16. doi: 10.1016/j. clae.2015.04.007. 